\title{
Zur Entstehung der überthermischen kosmischen Radiofrequenzstrahlung*
}

\author{
Von R. W. Larenz \\ Aus dem Institut für theoretische Physik der Technischen Hochschule Hannover \\ (Z. Naturforschg. 10 a, 901-913 [1955]; eingegangen am 2. September 1955)
}

\begin{abstract}
Im Anschluß an frühere Untersuchungen über mit Ladungstrennung verbundene Strömungen großer Amplitude im kompressiblen Plasma wird ein einfacher, auf dem Zusammenwirken von Plasmaströmungen beruhender Mechanismus für die Entstehung der ,gestörten“ Radiostrahlung ohne Zuhilfenahme statischer Magnetfelder angegeben, der die beobachteten Intensitäten ohne Schwierigkeiten zu erklären gestattet und das gelegentlich festgestellte Auftreten von Harmonischen der Plasmafrequenz verstehen läßt. Im Gegensatz zu bekannten Plasmawellentypen können die aus dem behandelten Mechanismus resultierenden Wellen sich mit der Plasmafrequenz bei endlichem Brechungsindex ausbreiten.
\end{abstract}

$\mathrm{I}$ $\mathrm{n}$ einer im folgenden mit A bezeichneten Arbeit ${ }^{1}$ war gezeigt worden, daß sich im Plasma zwei Formen adiabatisch reversibler, longitudinaler, schallwellenartiger Dichte- und Raumladungsschwankungen ausbilden können. Diese abkürzend als Ionenund Elektronenschall bezeichneten Plasmawellen breiten sich mit einer oberhalb der Ionenschallgeschwindigkeit bzw. der Elektronenschallgeschwindigkeit liegenden Fortpflanzungsgeschwindigkeit aus. Hierbei sind den Elektronenplasmawellen Frequenzen oberhalb der Plasmafrequenz zugeordnet, während Ionenschallwellen ins Gewicht fallender Ladungstrennung durch die Umgebung der um einen Faktor $\sqrt{m_{\mathrm{e}} / m_{\mathrm{i}}} \quad(m=$ Teilchenmasse $)$ unter der Elektronenplasmafrequenz liegenden Ionenplasmafrequenz gekennzeichnet sind. Zeichnen die radioastronomischen Beobachtungen einmal schon deutlich die Elektronenplasmafrequenz aus, so ergaben vor allem die Abschätzungen über die Größenordnung von Potentialdifferenzen und elektrischer Feldstärke in $\mathrm{A}$, daß als Ursache der Entstehung der überthermischen kosmischen Radiostrahlung im wesentlichen nur makroskopisch hydrodynamische Elektronenbewegungen verantwortlich gemacht werden können. Für die Zwecke der vorliegenden Arbeit sei daher nur eine Elektronendichteschwankung zugelassen und damit die Betrachtung auf das Gebiet des „Elektronenschalls" beschränkt.

Dichteschwankungen und Ladungstrennung müssen wir unbedingt zur Erklärung der kosmischen Radioemission heranziehen, zunächst einmal aus dem einfachen Analogiegrund, daß bei terrestrischen Radiosendern stets Raumladungseffekte im Spiel

* Vorgetragen auf der Tagung der Nordwestdeutschen Physikalischen Gesellschaft in Aachen am 23. 4. 1955.

1 R. W. L a r e n z, Z. Naturforschg. 10a, 766 [1955]. sind wie entsprechend auch beim Empfang und bei der Registrierung elektromagnetischer Strahlung, zweitens aber, weil es viel leichter zu verstehen ist, daß longitudinale Ladungsträgerbewegungen bei der Entstehung der Strahlung primär beteilig sind, da die Strahlung ihre Energie aus Gasströmungen beziehen muß, für die letztlich longitudinal wirkende Ursachen (Stoßwellen) sehr viel plausibler sind als reine, in sich geschlossene, raumladungsfreie Wirbelbewegungen mit schwer zu übersehendem Antriebsagens. Hinzu kommt, daß raumladungsfreie elektromagnetische Plasmawellen sich mit der Plasmafrequenz bekanntlich nicht ausbreiten können, da der Brechungsindex bei dieser Frequenz verschwindet. Plasmawellen, bei denen neben einem Transversalanteil ein longitudinaler auftritt, erhält man zwar schon, wie aus der Ionosphärentheorie bekannt, wenn sich elektromagnetische Wellen in einem statischen Magnetfeld ausbreiten, dessen Feldrichtung nicht mit der Wellenfortpflanzungsgeschwindigkeit zusammenfällt. Aber auch in diesem Fall, wo der Longitudinalanteil im allgemeinen nur die Rolle eines durch das Magnetfeld bedingten sekundären Effekts spielt ${ }^{2}$, ergibt sich bei der Plasmafrequenz ein Verschwinden oder sogar Imaginärwerden des Brechungsindex (solange, wie im Normalfall, die Larmorfrequenz nicht größer als die Plasmafrequenz ist). Dagegen gibt es einen sehr einfachen Strömungsmechanismus, der, wie wir in dieser Arbeit zeigen wollen, ohne Zuhilfenahme statischer Magnetfelder zu gekoppelten elektromagnetischen Transversal- und Longitudinalwellen führt, bei denen die Longitudinalbewegung den Primäreffekt darstellt

\footnotetext{
2 Zwischen Longitudinal- und Transversalanteil besteht eine Phasenverschiebung von $\pi / 2$; das Magnetfeld wirkt also reaktiv.
} 
und denen ein Brechungsindex zugeordnet ist, der für die in Frage kommenden Frequenzen Ausbreitung zuläßt.

\section{§ 1. Der Mechanismus}

Reine longitudinale Dichte- und Raumladungswellen in einem im Mittel ruhenden oder in Wellenrichtung strömenden Plasma sind bekanntlich nicht von einem Magnetfeld begleitet, da sich Leitungsund Verschiebungsstrom kompensieren. Sie können daher auch nicht zu elektromagnetischer Ausstrahlung führen. Dies ändert sich jedoch, wenn eine Raumladungswelle, die sich z. B. in der $x$-Richtung (s. Abb. 1) ausbreiten möge, überlagert wird durch eine allgemeine Plasmaströmung, deren Geschwindigkeit $\mathfrak{v}_{\mathrm{D}}$ mit der Ausbreitungsrichtung der Raumladungswelle einen endlichen Winkel $\vartheta$ einschließt.

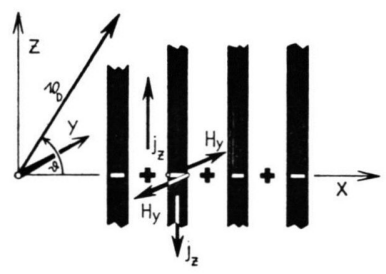

Abb. 1. Zur Veranschaulichung des Mechanismus.

Diese als „Driftbewegung“ bezeichnete Plasmaströmung braucht nicht von Dichteschwankungen begleitet zu sein und sie soll das Plasma als Ganzes, also Ionen und Elektronen in gleicher Weise erfassen. Die aufeinanderfolgenden positiven und negativen Raumladungen der Dichtewelle stellen für einen ortsfesten Beobachter einen Wechselstrom dar, der infolge der überlagerten Driftströmung nun eine zur Wellenfortpflanzungsrichtung transversale Komponente $j_{z}$ proportional $\left|\mathfrak{v}_{\mathrm{D}}\right| \sin \vartheta$ besitzt. Diese Transversalkomponente des Plasmastroms ist natürlich mit einem ebenfalls transversalen Magnetfeld $H_{y}$ verbunden, dessen Feldrichtung senkrecht zu der von Driftgeschwindigkeitsvektor und Wellengeschwindigkeitsvektor aufgespannten $x-z$-Ebene liegt. Da es sich um ein zeitlich sich änderndes magnetisches Feld handelt, wird ein elektrisches Wechselfeld $E_{z}$ parallel zur Richtung des ursprünglich als Konvektionsstrom zustandegekommenen Transversalstromes induziert. Das induzierte Feld wirkt auf die Transversalströmung zurück und es ergibt sich so eine gekoppelte elektromagnetische Longitudinal- und Transversal- welle. Eine weitere Kopplung zwischen den beiden Bewegungsrichtungen ergibt sich noch durch die Lorentz-Kraft. Da das magnetische Feld und die quellenfreie Komponente des elektrischen Feldes senkrecht zur Richtung der Dichtewellenfortpflanzung liegen, hat der aus beiden Feldvektoren gebildete Poyntingsche Vektor des Strahlungsstromes eine Richtung parallel zur Dichtewellenausbreitung. Von dieser Vorstellung ausgehend hat man also eine Ausstrahlung analog derjenigen einer Langdrahtantenne, für die (bei gegenüber der Wellenlänge hinreichend großer geometrischer Länge) Strahlungs. ausbreitung und Laufrichtung der Raumladungswellen zusammenfallen.

In den folgenden Paragraphen werden wir diese Vorstellung quantitativ behandeln, wobei wir bei dem einfachen Bild ebener, seitlich nicht begrenzter Dichtewellen bleiben, um die mathematischen Schwierigkeiten nicht größer als nötig werden zu lassen. Allgemein wird man immer ein elektromagnetisches Strahlungsfeld in ionisierten Gasen erhalten, wenn Raumladungsschwankungen mit beliebigen Strömungen zusammenwirken; mit anderen Worten: bei Vorliegen kompressibler Turbulenz.

\section{§ 2. Differentialgleichungen}

Wir legen die gleichen Voraussetzungen zugrunde wie bei der Elektronenschallbehandlung in der Arbeit A, also ein Plasma mit einem der adiabatischen Zustandsgleichung gehorchenden Elektronengas von der Ruhetemperatur $T_{0}$ und der mittleren Dichte $N_{0}$, die gleich der unveränderlichen Ionendichte ist. Die Grundgleichungen, die wir benötigen, Eulersche Bewegungsgleichung, Kontinuitätsgleichung und Maxwellsche Gleichungen, lauten daher zunächst:

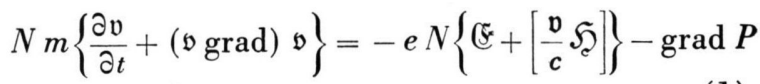

$$
\begin{aligned}
& \text { mit } \quad P=N_{0} k T_{0}\left(N / N_{0}\right)^{5 / s} \text {, } \\
& \frac{\partial N}{\partial t}+\operatorname{div} N \mathfrak{v}=0, \\
& \frac{e}{m c} \mathfrak{S}=\operatorname{rot} \mathfrak{Z}, \\
& \frac{e}{m c} \xi=-\frac{1}{c} \frac{\partial \mathscr{U}}{\partial t}-\operatorname{grad} \Phi, \\
& c^{2} \Delta \mathfrak{Z}-\frac{\partial \mathscr{2} \mathfrak{l}}{\partial t^{2}}=-\frac{4 \pi e}{m} j=\frac{\omega_{\mathrm{e}}^{2}}{N_{0}}\left(N \mathfrak{v}-N_{0} \mathfrak{b}_{\mathrm{D}}\right)
\end{aligned}
$$


mit $\omega_{\mathrm{e}}^{2}=\frac{4 \pi e^{2} N_{0}}{m}$

und $\mathfrak{v}_{\mathrm{D}}=$ Driftgeschwindigkeit des Plasmas,

$$
\operatorname{div}\left(\xi=4 \pi e\left(N_{0}-N\right)\right. \text {. }
$$

Wir berücksichtigen also entgegen der in der Plasmawellentheorie sonst üblichen Gepflogenheit die Lorentz-Kraft als Rückwirkung des Eigenmagnetfeldes der Plasmaströme. Ferner berücksichtigen wir den Einfluß des Elektronendrucks $P$, der, wie sich in A gezeigt hat, für die Begrenzung der Ladungstrennung maßgeblich ist. Nach der in $\S 1$ entworfenen Vorstellung werden wir Lösungen der hydrodynamischen Plasmagleichungen aufsuchen, bei denen die Zustands- und Feldgrößen nur von einer Raumkoordinate $x$ und der Zeit $t$ abhängen. Um auf gewöhnliche Differentialgleichungen zu kommen, werden wir entsprechend dem Verfahren, das sich in A bewährt hat, quasistationäre Strömungen untersuchen, für die alle Größen nur Funktionen vom Argument $x^{*}=x-v_{0} t$ sind, so daß ein mit der Geschwindigkeit $v_{0}$ in $x$-Richtung mit der Strömung mitbewegter Beobachter ein zeitlich nicht veränderliches Strömungsbild wahrnimmt ${ }^{3}$. Bei Transformation obiger Gleichungen auf das Koordinatensystem des mitbewegten Beobachters, Komponentenzerlegung der Vektorgleichungen und Ersetzung von (E) und $\mathfrak{S}$ durch $\mathfrak{Z}$ und $\Phi$ gehen diese Gleichungen in die folgenden über, wenn wir Ableitungen nach $x^{*}$ durch einen Strich kennzeichnen und die Orientierung des Koordinatensystems wie in $\S 1$ beibehalten, so daß die für den Vorgang wichtige Driftgeschwindigkeitskomponente $\left|\mathfrak{b}_{\mathrm{D}}\right| \sin \vartheta=v$ in die $z$-Richtung fällt.

$$
\begin{gathered}
v_{x} v_{x}{ }^{\prime}-c \Phi^{\prime}+\left(\bar{v}+v_{z}\right) A_{z}{ }^{\prime}+\frac{5 k T_{0}}{2 m}\left(\frac{N}{N_{0}}\right)^{2 / s^{\prime}}=0, \\
v_{x} v_{z}{ }^{\prime}-v_{x} A_{z}{ }^{\prime}=0,
\end{gathered}
$$

$\left(N v_{x}\right)^{\prime}=0$ oder gleich integriert $v_{x} / v_{0}=N_{0} / N$,

$$
\begin{gathered}
A_{z}^{\prime \prime}\left(1-\frac{v_{0}^{2}}{c^{2}}\right)=\frac{\omega_{\mathrm{e}}^{2}}{c^{2}}\left\{\frac{N}{N_{0}}\left(\bar{v}+v_{z}\right)-\bar{v}\right\}, \\
\Phi^{\prime \prime}=\frac{\omega_{\mathrm{e}}^{2}}{c}\left(N / N_{0}-1\right) .
\end{gathered}
$$

Dieses Differentialgleichungssystem, in dem die Geschwindigkeiten jetzt auf das mitbewegte Koordinatensystem bezogen sind, kann, wie wir im Rest dieses Paragraphen zeigen wollen, auf eine einzige für das Problem charakteristische Differentialgleichung zurückgeführt werden.

Gl. (8) läßt sich sofort integrieren zu

$$
A_{z}=v_{z},
$$

wobei eine Integrationskonstante dadurch berücksichtigt ist, daß wir die Elektronengeschwindigkeitskomponente in $z$-Richtung von vornherein in den konstanten Driftanteil $\bar{v}$ und den Wellenanteil $v_{z}$ zerlegt haben. $N, A_{z}$ und nach Differentiation von (7) auch $\Phi$ lassen sich leicht aus den Gleichungen eliminieren. Wir führen nun die dimensionslosen Variablen

$$
v_{x} / v_{0}=w, \quad v_{z} / \bar{v}=u, \quad \xi=2 \pi x^{*} / \lambda=x^{*} \omega / v_{0}
$$

ein und bezeichnen durch einen Strich in Zukunft Ableitungen nach $\xi$. Führen wir noch mit $n=c / v_{0}$ den Brechungsindex, mit

$$
a_{\mathrm{e}}=\sqrt{5 k T_{0} / 3 m}
$$

die Elektronenschallgeschwindigkeit ein und schließlich noch die Abkürzungen

$$
\omega_{\mathrm{e}} / \omega=\alpha, \quad \bar{v} / \boldsymbol{c}=\varepsilon \text { und }^{4} \quad a_{\mathrm{e}} / \boldsymbol{c}=\eta,
$$

so bleiben die gekoppelten Differentialgleichungen 2. Ordnung:

$$
\begin{gathered}
\frac{1}{2 \alpha^{2}}\left\{w^{2}+3 \eta^{2} n^{2} w^{-2 / 3}+\varepsilon^{2} n^{2}(1+u)^{2}\right\}^{\prime \prime}=\frac{1}{w}-1 \quad\left(\sim \Phi^{\prime \prime}\right), \\
\left(n^{2}-1\right) u^{\prime \prime} / \alpha^{2}=(1+u) / w-1 \quad\left(\sim A_{z}^{\prime \prime}\right) .
\end{gathered}
$$

Auf Grund der Identität $2 y^{\prime \prime} \equiv \mathrm{d}\left(y^{\prime 2}\right) / \mathrm{d} y$ läßt sich (14) auch schreiben

$$
w=\mathrm{d}(1+u)^{2} / \mathrm{d}\left\{\left(n^{2}-1\right) u^{\prime 2} / \alpha^{2}+2(1+u)\right\}
$$

und (13) läßt sich unter Berücksichtigung von (14) bzw. (14a) einmal integrieren zu

$$
\begin{aligned}
\frac{1}{4 \alpha^{2}}\left\{w^{2}+3 \eta^{2} n^{2} w^{-2 / 5}\right. & \left.+\varepsilon^{2} n^{2}(1+u)^{2}\right\}^{\prime 2} \quad\left(\sim \Phi^{\prime 2}\right) \\
& =\text { const }-(w-1)^{2}+3 \eta^{2} n^{2}\left\{\frac{2}{5} w^{-5 / s}-w^{-2 / 3}\right\}+\varepsilon^{2} n^{2}\left\{\frac{n^{2}-1}{\alpha^{2}} u^{\prime 2}+2(1+u)-(1+u)^{2}\right\} .
\end{aligned}
$$

3 Die Geschwindigkeit $v_{0}$ sei dabei auf das mit der Driftgeschwindigkeitskomponente $\left|\mathfrak{v}_{\mathrm{D}}\right| \cos \vartheta$ einheitlich in $x$-Rich-

tung strömende Medium bezogen; sie soll also diese für das Weitere unwichtige Komponente nicht enthalten.

${ }^{4} \eta^{2} n^{2} \equiv K_{\mathrm{e}}$ in Arbeit A. 
Hierin kann man $w$ nach (14) einsetzen und erhält dann eine Differentialgleichung dritter Ordnung für $u(\xi)$, die sich jedoch mit $(14 \mathrm{a})$ in eine Differentialgleichung zweiter Ordnung für $u^{\prime}(u)$ oder in den Variablen der rechten Seite von (14a) überführen läßt. Schreiben wir

$$
\zeta=(1+u)^{2}, \quad \varphi=\frac{n^{2}-1}{a^{2}} u^{\prime 2}+2(1+u),
$$

so lautet (14a) $w=\mathrm{d} \zeta / \mathrm{d} \varphi$, und (15) geht damit in die für das Problem charakteristische Differentialgleichung über

$$
\begin{aligned}
\left\{2 \frac{\mathrm{d}^{2} \zeta}{\mathrm{d} \varphi^{2}}\left[1-\eta^{2} n^{2}\left(\frac{\mathrm{d} \zeta}{\mathrm{d} \varphi}\right)^{-\delta / 3}\right]+\varepsilon^{2} n^{2}\right\}^{2} \frac{\zeta}{n^{2}-1}(\varphi & -2 \sqrt{\zeta}) \\
& =\text { const }-\left(\frac{\mathrm{d} \zeta}{\mathrm{d} \varphi}-1\right)^{2}+3 \eta^{2} n^{2}\left\{\frac{2}{5}\left(\frac{\mathrm{d} \zeta}{\mathrm{d} \varphi}\right)^{-5 / 3}-\left(\frac{\mathrm{d} \zeta}{\mathrm{d} \varphi}\right)^{-2 / 3}\right\}+\varepsilon^{2} n^{2}(\varphi-\zeta),
\end{aligned}
$$

nach deren Lösung nur noch eine Quadratur durchzuführen ist, um $u(\xi)$ zu erhalten ${ }^{5}$.

\section{$\S$ 3. Diskussion der Differentialgleichungen und spezieller Lösungen}

Die beiden nun folgenden Paragraphen beschäftigen sich mit der Lösung der vorstehenden komplizierten Differentialgleichung (16), enthalten also vorwiegend mathematische Überlegungen. Ihr Ziel ist es, das elektrodynamische Feld des zugrundeliegenden Strömungsvorganges und damit den Strahlungsstrom sowie die Dispersionsformel aufzufinden. Eine exakte Integration der Gl. (16) ist allgemein nicht durchführbar. Aus einer qualitativen Diskussion über das Verhalten der Lösungskurven und die Lösungen einiger Spezialfälle ergibt sich ein zweckmäßiger Ansatz zur näherungsweisen Lösung der Gleichung, der sie auf eine schon in Arbeit A behandelte und gelöste Gleichung zurückzuführen erlaubt.

Die linke Seite von (16) ist, wie ihre Herleitung zeigt, proportional dem Quadrat von $\Phi^{\prime}$. Es sind also nur solche Lösungen physikalisch sinnvoll, für die diese linke Seite $>0$ ist. Ferner muß $w=\mathrm{d} \zeta / \mathrm{d} \varphi$ stets $>0$ sein, da es umgekehrt proportional der Dichte ist. Betrachten wir also den Verlauf der Lösungskurven in einer $\zeta-\varphi$-Ebene (Abb. 2), so ergibt sich folgendes:

Die Extremwerte von $u$, für die $u^{\prime}=0$ gilt, liegen auf Grund der Definition von $\zeta$ und $\varphi$ auf der Parabel $\zeta=\varphi^{2} / 4$ oder $\varphi=2 \sqrt{\zeta}$, die also von den Lösungskurven geschnitten werden muß. Da nun auf dieser Parabel die linke Seite von (16) verschwindet, so sind diese Schnittpunkte gleichzeitig die Endpunkte physikalisch sinnvoller Lösungen;

5 Eine äquivalente, aber nicht weniger komplizierte, für das Problem charakteristische Differentialgleichung 2. Ordnung läßt sich für die Variablen $\Phi$ und $\Phi^{\prime}$ ableiten. und zwar verlaufen für $n^{2}>1$ bzw. $<1$ diese Lösungskurven unter bzw. über der Parabel. Durch die Bedingung $\Phi^{\prime 2}=0$ an den Endpunkten ist dann auch die Konstante auf der rechten Seite von (16) festgelegt. Da $w=\mathrm{d} \zeta / \mathrm{d} \varphi \sim 1 / N$ wie schon festgestellt stets $>0$ sein muß, verlaufen sämtliche Lösungen zwischen den Schnittpunkten mit der genannten Parabel monoton und es kommen nur $\varphi \geqq 0$ und $u \geqq-1$ zugeordnete Lösungen in Frage.

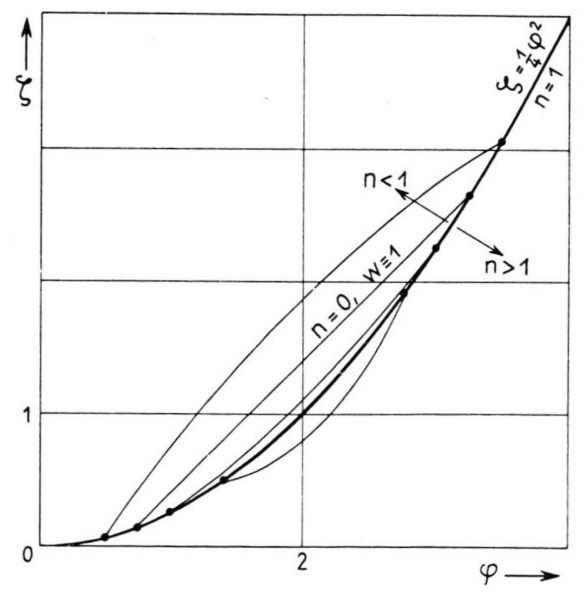

Abb. 2. Das Aussehen der Lösungskurven von Diff.-Gl. (16).

Damit für eine bestimmte Lösung eine eindeutige Zuordnung zwischen $u$ und $w$ möglich ist, muß auch $\mathrm{d} \zeta / \mathrm{d} \varphi$ eine monotone Funktion sein. Lösungen, die mit größerer Ladungstrennung bzw. Dichteschwankung verbunden sind, bei denen also $w=\mathrm{d} \zeta / \mathrm{d} \varphi$ stärker variiert, zeigen einen stärker gekrümmten Verlauf. Für $n^{2}>1$ sind nur Lösungen mit $\mathrm{d}^{2} \zeta / \mathrm{d} \varphi^{2}>1 / 2$ möglich, $w$ und $u$ sind in Phase. Für $n^{2}<1$ existieren Lösungen mit $1 / 2>\mathrm{d}^{2} \zeta / \mathrm{d} \varphi^{2} \lessgtr 0$, es gibt gleich- und gegenphasige Bewegungen für 
$w$ und $u$; der Grenzfall $\mathrm{d}^{2} \zeta / \mathrm{d} \varphi^{2}=0, w \equiv 1$ wird für $n^{2}=0$ erreicht, was einer Welle mit verschwindender Dichteschwankung entspricht.

Wir sind in erster Linie an Lösungen interessiert, die mit nennenswerten Dichte- und Raumladungsschwankungen verknüpft sind, die wir bei unserem Mechanismus als primäre Ursache der Strahlungsentstehung bezeichnet haben. Der Betrag der wahren longitudinalen Elektronengeschwindigkeitsamplitude ist durch

$$
v_{\mathrm{L}}=\left|v_{x \mathrm{~m}}-v_{0}\right|=\frac{c}{n}\left|w_{\mathrm{m}}-1\right|=\frac{c}{n} \mid \frac{N_{0}}{N_{\mathrm{m}}}-1
$$

gegeben. Sollen die hydrodynamischen Geschwindigkeiten in vertretbaren Grenzen bleiben, mindestens aber kleiner als die Lichtgeschwindigkeit $c$ sein, dann muß, wenn gleichzeitig stärkere Dichteschwankungen möglich. sein sollen, der Brechungsindex $n \gtrsim 1$ sein. Ist umgekehrt $n$ wesentlich kleiner als 1 , so können wir vom physikalischen Gesichtspunkt aus keine großen Dichte- und damit $w$-Schwankungen erwarten. Für diesen letzteren, uns hier weniger interessierenden Bereich würde es daher genügen, die Differentialgl. (13) und die mit $1 / w=1+\delta$ fast lineare Gl. (14) etwa mit einem zweigliedrigen Fourier-Ansatz näherungsweise zu lösen.

Für größere Dichte-Amplituden müssen wir die Ausgangsgleichungen in allgemeiner Form zu lösen versuchen. Die auch bei Fortlassung der Druckglieder $(\eta=0)$ komplizierte, nicht rationale Differentialgl. (16) läßt keine exakte Lösungsangabe in allgemeiner Form zu. Wir müssen daher zu Näherungsverfahren greifen. Das monotone Verhalten der Lösungskurven von (16) und ihrer Steigung legt es nahe, diese durch Parabelbögen mit $\mathrm{d}^{2} \zeta / \mathrm{d} \varphi^{2}=$ const anzunähern (etwa im Sinne des Ritzschen Verfahrens). Bevor wir dazu übergehen, wollen wir noch kurz spezielle Lösungen von (16) bzw. (13), (14) und (15) diskutieren, die ebenfalls eine Näherung der skizzierten Art für den allgemeinen Fall nahelegen.

I. Bei endlichem $u^{\prime \prime}$ und $\alpha$ ist bei $n^{2}=1$ Gl. (14) mit $1+u=w$ exakt erfüllt. Gl. (13) bzw. (15) nehmen dann bis auf konstante Faktoren die gleiche Form an, wie die aus der Arbeit A bekannten „Elektronenschall"-Gleichungen, deren Lösung durch Quadratur gewonnen werden und auch mit sehr guter Näherung durch einen einfachen analytischen Ausdruck angegeben werden kann (siehe auch $\S 4$ ).

II. In den Lösungsbereichen, für die

$$
u^{2} \ll|u| \ll|w-1|
$$

gilt, kann (14) näherungsweise geschrieben werden

$$
\frac{n^{2}-1}{\alpha^{2}} u^{\prime \prime} \simeq \frac{1}{w}-1 \quad\left(\sim \Phi^{\prime \prime} \sim A_{z}^{\prime \prime}\right),
$$

und in (13) kann $\varepsilon^{2} n^{2}(1+u)^{2 \prime \prime}$ durch

$$
2 \varepsilon^{2} n^{2} u^{\prime \prime} \simeq \frac{2 \varepsilon^{2} n^{2} \alpha^{2}}{n^{2}-1}\left(\frac{1}{w}-1\right)
$$

ersetzt werden. Gl. (13) nimmt dann bis auf konstante Koeffizienten wiederum die aus A bekannte Form mit der einfachen Integrationsmöglichkeit an. Wenn man die Rechnung durchführt, zeigt sich, daß obige Bedingung für $n^{2} \gg 1$ zutrifft (praktisch $n^{2} \geqq 3$, „langsame“ elektromagnetische Wellen mit schwacher Rückwirkung); wir haben hier also eine asymptotische Lösung vor uns.

In beiden hier untersuchten Fällen für $n^{2}=1$ und $n^{2} \gg 1$ ergibt sich die gleiche Lösungsform $w(\xi)$ wie in A; diese Lösungsform wird daher die exakte Lösung auch für die übrigen Wertebereiche von $n^{2}$ gut annähern. Wir erhalten diese Lösungsform stets, wenn wir $(1+u)^{2^{\prime \prime}}=w^{2^{\prime \prime}} / C$ mit $C=$ const setzen. Im folgenden Paragraphen werden wir sehen, daß ein solcher Ansatz gerade dem Vorschlag äquivalent ist, die Lösungskurven von (16) durch Parabelbögen anzunähern.

In Arbeit A haben wir den für die Bestimmung der maximal möglichen Ladungstrennung wichtigen Grenzfall untersucht, daß in Gl. (15) in Abhängigkeit von $w$ für

$$
w=w_{\mathrm{g}}=\left\{\eta^{2} n^{2} /\left(1+\varepsilon^{2} n^{2} \cdot \mathrm{d}(1+u)^{2} / \mathrm{d}\left(w^{2}\right)\right)\right\}^{3 / 8}
$$

links und rechts eine doppelte Nullstelle auftritt, was zur Ausbildung einer Dichtespitze mit unstetiger Ableitung führte. Dieser Fall kann hier niemals eintreten, wie man an der speziellen Lösung I für $n^{2}=1,1+u=w$ sofort erkennt, denn an der Spitze müßten wegen der unstetigen Ableitung von $u$ magnetisches Feld und die $z$-Komponente der elektrischen Feldstärke unstetig werden, was unmöglich ist. Könnte im allgemeinen Fall $\left(n^{2} \neq 1,1+u \neq w\right) w$ allein eine Spitze haben, so müßte dort $\mathrm{d} u / \mathrm{d} w=0, \mathrm{~d}^{2} \zeta / \mathrm{d} \varphi^{2}=\infty$ sein, was zu Widersprüchen führt, wenn man damit in die Diff.-Gl. (16) eingeht. $w$ kann sich dem Wert $w_{\text {g }}$ beliebig nähern, die Lorentz-Kraft sorgt aber dafür, daß er nicht angenommen wird.

\section{§4. Allgemeine Näherungslösung}

Mit dem durch die vorhergegangenen Untersuchungen nahegelegten Näherungsansatz

$$
\zeta=A+B \varphi+C \varphi^{2} / 4
$$

wird nun die Integration der Differentialgl. (16) durchgeführt. Die Lösung $w(\xi)$ kann dabei unmittelbar aus der Arbeit A übernommen werden, so daß 
die Rechnungen dieses Paragraphen in der Hauptsache die Bestimmung der Koeffizienten $A, B$ und $C$ unseres Ansatzes betreffen. Aus den Beziehungen zwischen den Koeffizienten ergibt sich zwanglos die Dispersionsformel (27). Aus der Lösung $u(\xi)$ werden dann die Feldgrößen $E_{z}$ und $H_{y}$ berechnet und damit der Ausdruck (30) für den Strahlungsstrom $S_{x}$ gefunden.
Aus unserem Ansatz (17) folgt

$$
w=\mathrm{d} \zeta / \mathrm{d} \varphi=B+C \varphi / 2
$$

und daher als Beziehung zwischen $\zeta=(1+u)^{2}$ und $w$

$$
(1+u)^{2}=A-B^{2} / C+w^{2} / C .
$$

Bei Differentiation nach $\xi$ resultiert der zweite in $\S 3$ nahegelegte Ansatz, der also mit dem ersten gleichwertig ist.

Mit diesem Ansatz hat man als Lösung von (13), wie in Arbeit A gezeigt,

$$
\begin{gathered}
\pm \alpha \xi=\int_{w_{1}}^{w} \frac{\left\{w\left(1+\varepsilon^{2} n^{2} / C\right)-\eta^{2} n^{2} w^{-5 / 3}\right\} \mathrm{d} w}{\sqrt{\left(1+\varepsilon^{2} n^{2} / C\right)\left\{\left(w_{1}-1\right)^{2}-(w-1)^{2}\right\}-3 \eta^{2} n^{2}\left\{\frac{2}{5} w_{1}^{-5 / 3}-w_{1}^{-2 / 3}-\frac{2}{5} w^{-5 / 3}+w^{-2 / 3}\right\}}} \\
\text { mit } w_{1}>w_{\mathrm{g}}=\left\{\eta^{2} n^{2} /\left(1+\varepsilon^{2} n^{2} / C\right)\right\}^{3 / 8}<1 .
\end{gathered}
$$

Wir haben darin bereits die bei der ersten Integration von (13) auftretende Integrationskonstante durch die untere Grenze $w_{1}$ des Integrationsintervalls von $w$ ausgedrückt. Diese untere Grenze, wie auch die äußerste obere Grenze $w_{2}>1$ bestimmen sich dadurch, daß der Nenner $\left(\sim \Phi^{\prime}\right)$ im Integral $(20)$ verschwindet. Die untere Grenze $w_{1}$ kann den angegebenen Wert $w_{\mathrm{g}}$, für den auch der Zähler Null wird, nicht unterschreiten. Wie in Arbeit A ausgeführt, handelt es sich dabei um eine (praktisch nur bei $n \rightarrow 1 / \eta>1$, d. h. $v_{0} \rightarrow a_{\mathrm{e}}$ wirksame) Grenze, die der Druckeinfluß der Ladungstrennung setzt. (Siehe auch Schluß von § 3.) Das Integral läßt sich, wie in A näher besprochen, mit sehr guter Näherung durch einen analytischen Ausdruck darstellen, wenn man Zähler und Nenner bis zu Gliedern zweiter Ordnung in $w-1$ entwickelt, wodurch keine wesentliche weitere Beschränkung (mathematischer Natur) für die Amplituden von $w$ eintritt.

Es ergibt sich

$$
\begin{aligned}
& \alpha \sqrt{1+\frac{\varepsilon^{2} n^{2}}{C}-\eta^{2} n^{2}}\left(\xi-\frac{\pi}{2}\right) \\
& \simeq\left\{1+\frac{\varepsilon^{2} n^{2}}{C}-\eta^{2} n^{2}\left(1+\frac{10}{9}\left(w_{1}-1\right)^{2}\right)\right\} \arcsin \frac{w-1}{\left|w_{1}-1\right|} \\
&-\sqrt{\left(w_{1}-1\right)^{2}-(w-1)^{2}} \\
& \cdot\left\{1+\frac{\varepsilon^{2} n^{2}}{C}+\frac{5}{3} \eta^{2} n^{2}-\frac{10}{9} \eta^{2} n^{2}(w-1)\right\}
\end{aligned}
$$

mit $w_{1}>w_{\mathrm{g}}$.

Für vernachlässigbaren Druckeinfluß $(\eta n \rightarrow 0)$ ist (21) die strenge Lösung von (20).
Um nun $u(\xi)$ angeben zu können, müssen noch die Koeffizienten $A, B, C$ des Ansatzes (17) bestimmt werden. Gl. (21) entnimmt man, daß im Rahmen der hier durchgeführten Näherung für die Grenzamplituden $w_{1}, w_{2}$ die Beziehung

$$
1-w_{1}=w_{2}-1 \text { oder } w_{1}+w_{2}=2
$$

besteht. Andererseits gilt nach (18) für die Grenzwerte $w_{1,2}$ und $\varphi_{1,2}=2\left(1+u_{1,2}\right)$ bei $u^{\prime}=0$

$$
w_{1}+w_{2}=2 B+C\left(2+u_{1}+u_{2}\right)
$$

und

$$
w_{2}-w_{1}=C\left(u_{2}-u_{1}\right) \text {. }
$$

Schließlich muß der Ansatz (17) nach dem im $\S 3$ Gesagten an den Grenzen der Bedingung $\zeta=\varphi^{2} / 4$ genügen, woraus folgt, daß

$$
(1-C)\left(2+u_{1}+u_{2}\right)=2 B
$$

sein muß. Alle diese Beziehungen sind nur miteinander verträglich, wenn gilt

$$
\begin{gathered}
u_{1}=-u_{2}=\left(w_{1}-1\right) / C=\left(1-w_{2}\right) / C, \\
\left|u_{1}\right|=\left|u_{2}\right|=u_{\mathrm{m}}=\left|\left(w_{1}-1\right) / C\right|
\end{gathered}
$$

und

$$
B=1-C \text {. }
$$

1/C gibt also das Verhältnis der Amplitude von $u$ zur $w$-Schwankung an, d. h. also das Verhältnis der normierten Transversal- und Longitudinalgeschwindigkeitsamplitude.

Mit (22) und (23) folgt aus (19)

$$
u_{\mathrm{m}}^{2}=1+\frac{A}{1-C}=\left|\left(w_{1}-1\right) / C\right|^{2} .
$$

Eine weitere Beziehung zwischen den Koeffizienten ergibt sich, wenn wir fordern, daß die Diff.-Gl. (16) 
außer an den Enden des Gültigkeitsbereiches noch in einem $Z$ wischenpunkt streng erfüllt sei. Als eine solche mittlere Stelle wählen wir $w=\mathrm{d} \zeta / \mathrm{d} \varphi=1$, was der mittleren Dichte $N=N_{0}$ entspricht, um die die Schwankungen erfolgen.

Für $w=1$ ist

$$
\zeta=2+A-C=1+\left(w_{1}-1\right)^{2}(1-C) / C^{2}
$$

und $\varphi=2$

$$
\left(u_{(w=1)}=\frac{1-n^{2}}{2 \alpha^{2}} u_{(w=1)}^{\prime 2}\right) .
$$

Wenn wir bei Berechnung der Konstanten in (16) die mit gebrochenem Exponenten behafteten Druckglieder konsequenterweise wie bei der analytischen Darstellung des Integrals (20) bis zu Gliedern zweiter Ordnung in $w-1$ entwickeln, ergibt sich nach einiger Rechnung:

$$
\left.\frac{C-1}{n^{2}-1}=\frac{1+\sqrt{1+\left(\frac{w_{1}-1}{C}\right)^{2}(1-C)}}{2\left\{1+\left(\frac{w_{1}-1}{C}\right)^{2}(1-C)\right\}\left\{1+\frac{\varepsilon^{2} n^{2}}{C}-\eta^{2} n^{2}\right.}\right\} .
$$

Setzt man in (21) den zu $\xi=0$ gehörigen Wert $w=w_{1}$ oder $w=w_{.2}$ zu $\xi=\pi$ ein, so entsteht die Beziehung

$$
\begin{aligned}
\alpha \sqrt{1+\frac{\varepsilon^{2} n^{2}}{C}-\eta^{2} n^{2}} & \\
= & 1+\frac{\varepsilon^{2} n^{2}}{C}-\eta^{2} n^{2}\left\{1+\frac{10}{9}\left(w_{1}-1\right)^{2}\right\} .
\end{aligned}
$$

Gln. (25) und (26) bestimmen die Konstante $C$ als Funktion von $\alpha, n$ und der Amplitude

$$
\left|w_{1}-1\right|=\left|C u_{\mathrm{m}}\right| \text {. }
$$

Die beiden Beziehungen stellen gleichzeitig die amplitudenabhängige Dispersionsformel dar. Man sieht, $\mathrm{da} ß C \simeq n^{2}$ für $\varepsilon^{2} n^{2} / C-\eta^{2} n^{2} \ll 1$ ist. Für kleine Amplituden führen (25) und (26) zu

$$
\begin{gathered}
\left\{1+\varepsilon^{2} n^{2} / C-\eta^{2} n^{2}\right\} \cdot(C-1) /\left(n^{2}-1\right)=1, \\
\alpha^{2}=1+\varepsilon^{2} n^{2} / C-\eta^{2} n^{2},
\end{gathered}
$$

und damit ergibt sich die Dispersionsformel

$$
C=1+\left(n^{2}-1\right) / \alpha^{2}=\varepsilon^{2} n^{2} /\left(\alpha^{2}-1+\eta^{2} n^{2}\right)
$$

mit

$$
\alpha=\omega_{\mathrm{e}} / \omega,
$$

die man natürlich auch finden würde, wenn man die Ausgangsgleichungen für kleine Amplituden linearisiert, denn unsere für beliebige Amplituden aufgestellte Näherung muß selbstverständlich in die hierfür gültige lineare Näherung übergehen.

Neben der Lösung $w(\xi)$ nach (21) ist nun

$$
\begin{aligned}
u(\xi) & =u(w(\xi)) \\
& =\sqrt{1+\left(\frac{w_{1}-1}{C}\right)^{2}(1-C)+\frac{w^{2}(\xi)-1}{C}-1 .}
\end{aligned}
$$

Die damit ermittelte allgemeine Näherungslösung für $u$ enthält natürlich auch die im $\S 3$ besprochenen speziellen Lösungen, wie man dies unschwer am Beispiel $n^{2}=1$ erkennt. Gl. (25) verlangt hier $C=1$, womit $(28) 1+u \equiv w$ liefert.

Diese letztere Lösung ist dadurch ausgezeichnet, daß Dichte- und Transversalgeschwindigkeitsschwankungen so erfolgen, daß der resultierende transversale Plasmastrom gerade Null wird. Es handelt sich also um eine „Pseudovakuumwelle“ mit $\left|H_{y}(\xi)\right|=\left|E_{z}(\xi)\right|$.

Zur Berechnung der elektromagnetischen Strahlung interessieren uns die Feldgrößen

$$
E_{z}=-\frac{m}{e} \frac{\partial A_{z}}{\partial t}=\frac{m}{e} \omega \bar{v} u^{\prime} \quad \text { und } \quad H_{y}=-\frac{m c}{e} \frac{\partial A_{z}}{\partial x}=-\frac{m}{e} n \omega \bar{v} u^{\prime} .
$$

Da mit Gl. (20) $w^{\prime}(\xi)$ bekannt ist, ergibt sich

$$
u^{\prime}(\xi)=\frac{w^{2}}{2 C(1+u)}= \pm \alpha \frac{\left|w_{1}-1\right|}{C} \frac{\sqrt{1+\frac{\varepsilon^{2} n^{2}}{C}-\eta^{2} n^{2}} \sqrt{1-\left(\frac{w(\xi)-1}{w_{1}-1}\right)^{2}}}{\left\{1+\frac{\varepsilon^{2} n^{2}}{C}-\eta^{2} n^{2} w(\xi)^{-8 / 3}\right\} \sqrt{1+\left(\frac{w_{1}-1}{C}\right)^{2}(1-C)+\frac{w^{2}(\xi)-1}{C}}}
$$

mit $w(\xi)>w_{\mathrm{g}}$ nach Gl. (21).

Darin kann auch das Druckglied $w^{-8 / 3}$ noch entwickelt werden, wie wir das bereits im Zähler getan haben. Für $\eta^{2} n^{2}<1 / 10$ sind die mit diesem Faktor behafteten Glieder praktisch bedeutungslos, sofern nicht zu große Elektronenverdichtungen $d=1 / w_{1}$ betrachtet werden, die in die Nähe der in (20) an- gegebenen unteren Schranke $w_{\mathrm{g}}$ von $w_{1}$ fallen. Abgesehen von dieser Begrenzung ist bei der Wahl der Amplituden zu beachten, daß neben $\left|w_{1}-1\right|$ auch $u_{\mathrm{m}}=\left|\left(w_{1}-1\right) / C\right|$ stets kleiner als 1 sein müssen, $(u>-1$ nach $\S 3)$; weiter muß natürlich die wahre hydrodynamische Geschwindigkeitsamplitude der 
Elektronen $v_{\mathrm{L}}=(c / n)\left|w_{1}-1\right|$ kleiner als die Lichtgeschwindigkeit $c$ sein. Der Bereich der zweiten speziellen Lösung von $\S 3\left(n^{2}>3\right)$ ist dadurch gekennzeichnet, daß die Wurzel im Nenner von (29) mit wachsendem $n$ sich immer weniger von 1 unterscheidet.

Das Maximum von $u^{\prime}$ liegt in der Umgebung von $w=1$ (wenn sich $w$ dem Wert $w_{\text {g }}$ nicht zu sehr nähert). Setzen wir also zur Berechnung des Strahlungsstromes

$$
S_{x}=\frac{c}{4 \pi}\left[\mathfrak{E}, S_{\mathfrak{g}}\right]_{x}^{t}=-\frac{c}{4 \pi} \bar{E}_{z} H_{y}^{t},
$$

in (29) $w=1$ und beachten (26) bzw. (26a) sowie $1+\left[\left(w_{1}-1\right) / C\right]^{2}(1-C) \simeq 1$, so ergibt sich hierfür etwa der Betrag

$$
\begin{aligned}
S_{x}=\frac{m^{2} \omega^{2}}{8 \pi e^{2}} v^{2} \operatorname{cn}\left(\frac{w_{1}-1}{C}\right)^{2} & =N_{0} \frac{m}{2} \frac{\omega^{2}-v_{\mathrm{e}}^{2}}{\omega^{2}} \frac{c n}{C^{2}}\left(1-\frac{1}{d}\right)^{2} \\
& =N_{0} \frac{m}{2} \frac{\bar{v}^{2} v_{\mathrm{L}}{ }^{2}}{c} \frac{n^{3}}{C^{2} \alpha^{2}}, \quad(30)
\end{aligned}
$$

wenn $d$ die maximale Elektronenverdichtung bezeichnet. Ein Beispiel für Elektronendichteverteilung und elektrisches bzw. magnetisches Feld in der Welle ist in Abb. 3 wiedergegeben.

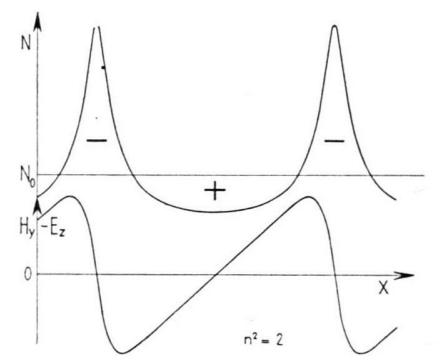

Abb. 3. Elektronendichte und elektromagnetische Feldgrößen als Funktion des Ortes in der Welle.

Der Poyntingsche Vektor besitzt neben dem Strahlungs. strom $S_{x}$ noch eine Komponente in $z$-Richtung, die proportional zu $\Phi^{\prime} H_{y}$ ist. Diese Komponente hat jedoch im Fall seitlich unbegrenzter ebener Wellen physikalisch keine Bedeutung, da sie zwar zeitabhängig, aber divergenzfrei ist $(\partial / \partial z \equiv 0) ;$ sie entspricht dem Feld des Poyntingschen Vektors, das einer Anordnung aus ruhenden Ladungen und Permanentmagneten zugeordnet ist. Elektromagnetische Energie kann nur durch ein divergenzbehaftetes [E్ $\mathfrak{F}]$-Feld übertragen werden.

Die erforderliche Nachprüfung, welches Vertrauen man in unsere mit den Gln. (20) bis (29) gewonnene Näherungslösung setzen kann, erfolgt zweckmäßig an Hand der Gl.(14). Schreiben wir

$$
\frac{n^{2}-1}{a^{2}} u^{\prime \prime}-\frac{1+u}{w}+1=F,
$$

worin $F$ bei exakter Gleichungserfüllung Null sein sollte, und tragen darin unsere im wesentlichen auf der Integration von (13) aufbauenden Näherungsausdrücke ein, so ergibt sich $F$ als Funktion von $w$ in Form eines länglichen Ausdrucks, auf dessen Anschreiben wir hier verzichten. Die Größe von $F$ im Vergleich zu den auf der linken Seite stehenden Termen ist dann ein Maß für den Grad der Genauigkeit unserer Lösung. Wir haben diese Prüfung durchgeführt im Gebiet größter Abweichung von den speziellen Lösungen für $n^{2}=1$ und $n^{2} \gg 1$, was in der Gegend von $n^{2}=2 \mathrm{zu}$ erwarten ist. Wir haben dabei eine der Amplitude $\left|w_{1}-1\right|=0,6$ (Verdichtung $d=2,5$ entsprechend Abb.3) hier zugeordnete hydrodynamische Geschwindigkeit von $0,6 c / \sqrt{2}$ zugrundegelegt und den Druckeinfluß vernachlässigt $\left(\eta^{2} n^{2}=\left(a_{\mathrm{e}} / v_{0}\right)^{2} \ll 1\right.$ für Elektronentemperaturen unter $\left.10^{8}{ }^{\circ} \mathrm{K}\right)$. Es ergab sich bei dieser extremen Geschwindigkeitsamplitude $F$ dem Betrage nach im Mittel kleiner als 5\% der Summe der Glieder eines Vorzeichens der linken Seite von (14b) und ferner war die Gleichung an zwei günstig gelegenen Stellen exakt erfüllt etwa derart, als ob die Koeffizienten $A, B, C$ des Ansatzes (17) durch eine Fehlerquadratmethode bestimmt worden wären.

Der Ansatz (17) stellt wegen der Bedeutung von $\varphi$ eine Differentialgleichung erster Ordnung für $u(\xi)$ dar, die durch elliptische Integrale gelöst werden kann. Die daraus resultierenden Funktionen erweisen sich aber gegenüber unseren mehr im Anschluß an Gl. (13) abgeleiteten Lösungen jedenfalls im hier hauptsächlich interessierenden Gebiet nennenswerter Ladungstrennung als schlechtere Näherung.

\section{§ 5. Die Dispersionsformel}

Nachdem nun mit der Lösung der Differentialgleichungen der vorwiegend mathematische Teil der Arbeit abgeschlossen ist, soll jetzt die Untersuchung der Ausbreitungsmöglichkeiten der bei unserem Strömungsproblem auftretenden elektromagnetischen Wellen folgen.

Gl. (27) läßt sich leicht nach $n^{2}$ oder $\alpha^{2}$ auflösen und man erhält

$$
\begin{aligned}
n^{2} & =\frac{1}{2 \eta^{2}}\left[\left(1-\varepsilon^{2}+\eta^{2}\right)\left(1-\alpha^{2}\right)+\varepsilon^{2}\right. \\
& \left. \pm \sqrt{\left\{\left(1-\varepsilon^{2}+\eta^{2}\right)\left(1-\alpha^{2}\right)+\varepsilon^{2}\right\}^{2}-4 \eta^{2}\left(1-\alpha^{2}\right)^{2}}\right] \\
\alpha^{2}= & \frac{\omega_{\mathrm{e}}^{2}}{\omega^{2}}=1-\frac{n^{2}}{2}\left(1-\varepsilon^{2}+\eta^{2}\right)+\varepsilon^{2} \\
& \pm \frac{n}{2} \sqrt{n^{2}\left(1+\varepsilon^{2}-\eta^{2}\right)^{2}-4 \varepsilon^{2}\left(n^{2}-1\right)} .
\end{aligned}
$$

Die Eigenschaften der Dispersionsformel übersieht man an Hand der qualitativen Wiedergabe in Abb. 4, wo $n^{2}$ über $\alpha^{2}=\omega_{\mathrm{e}}^{2} / \omega^{2}$ entsprechend der in der Ionosphärentheorie üblichen Darstellungsweise aufgetragen ist. Es fällt zunächst der schleifenartige Charakter ins Auge, der in ähnlicher Form bisher 


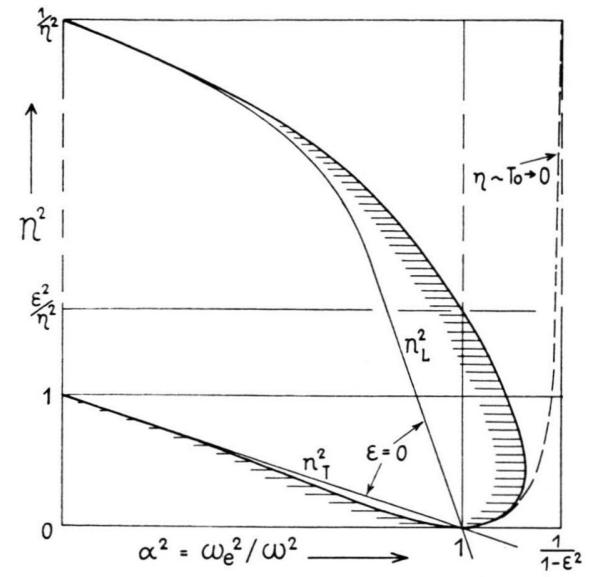

Abb. 4. Die Dispersionskurve. Die Schraffierung deutet die Verlagerung bei endlicher Amplitude an. $n_{\mathrm{T}}, n_{\mathrm{L}}=$ Brechungsindex reiner Transversal- bzw. Longitudinal-Wellen.

bei Plasmawellenuntersuchungen nicht in Erscheinung getreten ist. Diese Schleifenform ergibt sich als Folge der Kopplung zwischen transversaler und longitudinaler Wellenbewegung durch die Driftgeschwindigkeit. Im Grenzfall verschwindender Plasmadrift $(\varepsilon=\bar{v} / c \rightarrow 0)$ resultieren die bekannten, voneinander unabhängigen Dispersionsformeln für gewöhnliche elektromagnetische Wellen

$$
n_{\mathrm{T}}^{2}=1-\omega_{\mathrm{e}}^{2} / \omega
$$

und reine "Elektronenschallwellen“

$$
n_{\mathrm{L}}{ }^{2}=\frac{1}{\eta^{2}}\left(1-\frac{\omega_{\mathrm{e}}{ }^{2}}{\omega^{2}}\right)
$$

(siehe Arbeit A), die in der Abbildung mit eingezeichnet sind. Im Grenzfall verschwindenden Temperatur- und Druckeinflusses

$$
\eta^{2}=a_{\mathrm{e}}{ }^{2} / c^{2}=5 k T_{0} / 3 m c^{2} \rightarrow 0
$$

schwenkt der "Schallwellenast" in die für $T=0$ gezeichnete Lage ${ }^{6}$. Durch nähere Untersuchung der Gln. (25) und (26) findet man, daß sich der Kurvenzug bei endlicher Amplitude in Richtung des schraffierten Gebietes verlagert; d. h. wachsende Amplitude führt zu Frequenzsteigerung oder zu Erhöhung der Wellengeschwindigkeit bis auf das $\mathrm{Ge}$ biet anomaler Dispersion

$$
\mathrm{d} n / \mathrm{d} \alpha>0 \text { bei } \alpha=\omega_{\mathrm{e}} / \omega>1,
$$

wo Geschwindigkeitsabnahme eintritt.

Die Driftgeschwindigkeit $\bar{v}$ und die Elektronenschallgeschwindigkeit $a_{\mathrm{e}}$ werden im allgemeinen sehr

${ }^{6}$ Siehe hierzu auch R. W. La renz, Naturwiss. 40, 527 [1953]. klein sein gegen die Lichtgeschwindigkeit, so daß $\varepsilon=\bar{v} / c$ sowie $\eta=a_{\mathrm{e}} / c$ und erst recht $\varepsilon^{2}, \eta^{2}$ gegenüber 1 sehr kleine Zahlen sind. Dies führt dazu, daß der obere Zweig der Dispersionsformel im Gebiet $\alpha \sim 1, \omega \sim \omega_{\mathrm{e}}$ außerordentlich steil verläuft, d. h. ein Wellenzug kann sich mit von der Plasmafrequenz kaum abweichender Frequenz bei in weiten Grenzen beliebiger Wellengeschwindigkeit fortpflanzen. Dieser Sachverhalt läßt auch die Interpretation $\mathrm{zu}$, daß der Wert von $\varepsilon$, d. h. die Driftgeschwindigkeit bzw. deren transversale Komponente $\bar{v}$ unbedenklich örtlichen Schwankungen unterliegen kann, wie es im allgemeinen in den realen Radiostrahlungsplasmen der Fall sein wird, ohne daß dadurch die Fortpflanzungsbedingungen für die Plasmafrequenz wesentlichen Änderungen ausgesetzt wären. Die Ausdehnung des steil verlaufenden Teiles bei $\alpha \sim 1$ nimmt mit steigendem $\varepsilon / \eta \mathrm{zu}$; d. h. je größer das Verhältnis von Drift- zu Elektronenschall-Geschwindigkeit ist, desto ungestörter kann sich die Plasmafrequenz ausbreiten.

Bei der Diskussion der Differentialgleichungen in $\S 3$ haben wir bereits ausgeführt, daß für stärkere Dichtescliwankungen, die wir als primäre Ursache der Radiostrahlung ansehen, der Brechungsindex $n \gtrsim 1$ sein muß. Dies trifft für den oberen (Schallwellen-) Ast der Dispersionsformel zu und wir werden ihn dementsprechend als bei der Strahlungserzeugung maßgeblichen Zweig mit dem Namen „Entstehungsast“ belegen. Hingegen spielt der untere Zweig, der nahe dem Verlauf von $n^{2}$ für gewöhnliche Transversalwellen liegt, die Rolle des „Ausbreitungsastes“. Diese Unterscheidung wird deutlich, wenn wir das Amplitudenverhältnis der wahren Geschwindigkeitskomponenten $v_{z}$ und $v_{x}-v_{0}$ der Elektronen untersuchen, welches durch

$$
\frac{v_{\mathrm{T}}}{v_{\mathrm{L}}}=\frac{v_{z \mathrm{~m}}}{v_{x \mathrm{~m}}-v_{0}}=\frac{\bar{v} u_{\mathrm{m}}}{v_{0}\left(w_{1}-1\right)}=\frac{\bar{v}}{v_{0} C}=\frac{\varepsilon n}{C}
$$

gegeben ist. Drücken wir darin $C$ in erster Näherung nach (27) mit Hilfe der Dispersionsformel (31) als Funktion von $n^{2}$ aus, dann zeigt das Amplitudenverhältnis

$$
\begin{array}{r}
\frac{v_{\mathrm{T}}}{v_{\mathrm{L}}}=\frac{C(n)}{\varepsilon n}=\frac{1}{2 \varepsilon}\left[ \pm \sqrt{n^{2}\left(1+\varepsilon^{2}-\eta^{2}\right)^{2}-4 \varepsilon^{2}\left(n^{2}-1\right)}\right. \\
\left.-n\left(1-\varepsilon^{2}-\eta^{2}\right)\right]
\end{array}
$$

einen Verlauf, wie er qualitativ in Abb. 5 wiedergegeben ist.

Für den oberen Ast, für den $n^{2}>1$ sein kann, erweist sich die Geschwindigkeitsamplitude der 
Longitudinalbewegung und damit die kinetische Energie derselben, abgesehen von der unmittelbaren Umgebung von $n=0$, als stets beträchtlich größer als Amplitude und Energie der transversalen Wellenbewegung, während die Dinge auf dem unteren Ast umgekehrt liegen. Ein solches Überwiegen der longitudinalen Strömungsenergie auf dem Entstehungsast mußten wir vom energetischen Gesichtspunkt erwarten, wenn wir unsere Ansicht von der primären Bedeutung der Dichtewellen bei der Entstehung der Radiostrahlung bestätigt sehen wollten.

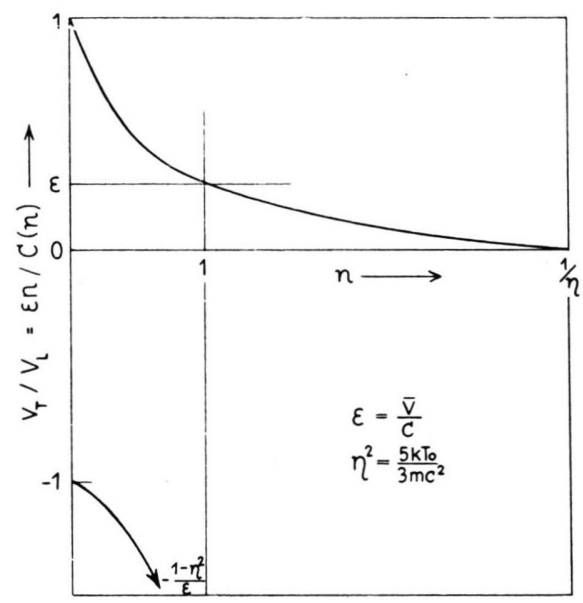

Abb. 5. Verhältnis von transversaler zu longitudinaler Geschwindigkeitsamplitude.

Geht die Erzeugung der Wellen mit etwa der Plasmafrequenz auf dem steil verlaufenden Teil des Entstehungsastes vor sich, so müssen sie später längs des Ausbreitungsastes verlaufen, um als reine elektromagnetische Transversalwellen in den Weltenraum hinauszulaufen. Der untere Ast ist dabei also so zu verstehen, daß entsprechend der in der Ionosphärentheorie ${ }^{7}$ üblichen Interpretation der Dispersionsformeln des Plasmas $\alpha=\omega_{\mathrm{e}} / \omega$ sich bei fester Frequenz $\omega$ (am Entstehungsort $\simeq \omega_{\mathrm{e}}$ entsprechend der dort herrschenden Dichte $N_{0}$ ) dadurch ändert, daß die Wellen ein Gebiet abnehmender Dichte $\sim \omega_{\mathrm{e}}{ }^{2}$ durchlaufen, wobei der Brechungsindex langsam gegen Eins geht. Um als gewöhnliche elektromagnetische Wellen in Erscheinung zu treten, müssen die Wellen also ihre longitudinalen Anteile „abstreifen", was offenbar in der Umgebung von $n \simeq 0$

7 Siehe etwa K. R a w e r, Die Ionosphäre, Groningen 1953, oder L. B ergmann u. H. L a s s en, Lehrbuch der drahtlosen Nachrichtentechnik, Bd. II, Berlin 1940.

8 Das Umspringen $\mathrm{d}{ }_{3}$ Amplitudenverhältnisses von +1 nach -1 bei $n=0, a=1$ geht bei Vorhandensein einer geschieht. Eine solche stetige Änderung des Verhältnisses von Transversal- zu Longitudinalanteil ist aus der Wellentheorie des Plasmas im Magnetfeld (Erdfeld) wohlbekannt ${ }^{7}$. Kritisch ist dabei stets die Umgebung von $n \rightarrow 0$, die für genauere Untersuchungen die Behandlung der Ausbreitung im inhomogenen Medium wegen der veränderlichen Dichte $N_{0}$ erfordert, da $n \rightarrow 0$ ja bedeutet, daß die Wellenlänge sehr groß wird, so daß sich schließlich merkliche Dichteänderungen innerhalb einer Wellenlänge ergeben ${ }^{8}$.

In Abhängigkeit von $\alpha$ nimmt das Verhältnis von transversaler zu longitudinaler Geschwindigkeitsamplitude längs des oberen Astes außerordentlich schnell $\mathrm{ab}$, wenn $\alpha$ von $\simeq 1$ nach 0 geht. Dies liegt daran, daß wegen des schon erwähnten steilen Verlaufs der Dispersionsformel bei $\alpha \simeq 1$ einer kleinen Umgebung von $\alpha=1$ ein großer Wertebereich von $n$ zugeordnet ist. Bei wesentlicher Entfernung von $\alpha=1$ wird mit $\eta n \rightarrow 1$ außerdem die in Arbeit A besprochene Amplitudenbegrenzung wirksam. Beide Sachverhalte begünstigen daher ein gegenüber höheren Frequenzen stark bevorzugtes Auftreten der Plasmafrequenz.

Aus dem steilen Verlauf der Dispersionskurve dürfen nicht ohne weiteres irgendwelche Schlüsse über die Gruppengeschwindigkeit von Wellenpaketen gezogen werden, da diesem Begriff, wie schon in A bemerkt, zumindest in der Form $v_{0}-\lambda \mathrm{d} v_{0} / \mathrm{d} \lambda$ in einer nichtlinearen Theorie keinerlei Bedeutung zukommt.

\section{§ 6. Der Strahlungsstrom}

Wie man der letzten Schreibweise des Ausdrucks (30) für den Strahlungsstrom entnimmt, ist $S_{x}$ proportional dem Quadrat des Produktes aus Driftund Dichteströmungsgeschwindigkeit, wobei der Proportionalitätsfaktor $n^{3} / C^{2} \alpha^{2}$ allerdings selbst noch von $\bar{v}$ und $v_{\mathrm{L}}$ abhängt. Seien nun $\bar{v}$ und $v_{\mathrm{L}}$ irgendwelche fest vorgegebenen Geschwindigkeiten, so hat $S_{x}$ mit der Funktion $n^{3} / C^{2} \alpha^{2}$ ein Maximum bei einem zum „Entstehungsast“ gehörigen Argument

$$
n \simeq 2 \varepsilon / \sqrt{3},
$$

wie man mit Hilfe von (27), (31) und (32) leicht findet, wenn man berücksichtigt, daß $\varepsilon^{2}$ und $\eta^{2}$ ge-

Dämpfung infolge endlicher Leitfähigkeit des Plasmas in einen stetigen Gang der Phasenlage des Amplitudenverhältnisses über, wobei gleichzeitig der Realteil des nunmehr komplexen Brechungsindex $n$ nicht mehr auf Null absinkt. 
gen 1 sehr kleine Zahlen sind. An dieser wegen $\varepsilon=\bar{v} / c \ll 1$ sehr nahe bei $n=0$ gelegenen Stelle ist die kinetische Energie der Longitudinalbewegung noch um einen Faktor $(\varepsilon n / C)^{2} \simeq 3$ größer als die der transversalen Wellenbewegung, und die Funktion $n^{3} / C^{2} \alpha^{2}$ hat hier den Wert $\simeq 2 / 3 \sqrt{3} \varepsilon$. Bei Vorgabe der Geschwindigkeiten $v_{\mathrm{L}}$ und $\bar{v}$ existiert also eine leicht angebbare obere Grenze für den möglichen Strahlungsstrom.

Von den Longitudinalgeschwindigkeiten $v_{\mathrm{L}}$ können wir annehmen, daß sie von gleicher Größenordnung wie die Driftgeschwindigkeiten $\bar{v}$ sind. Bei einer Reihe von intensiven kosmischen Radiostrahlungswellen und bei solaren Strahlungsausbrüchen hat man die Existenz von Strömungsgeschwindigkeiten von der Größenordnung $1000 \mathrm{~km} / \mathrm{sec}$ festgestellt ${ }^{9,10}$. Das sind Werte von der Größenordnung der Elektronenschallgeschwindigkeit

$$
a_{\mathrm{e}}=\sqrt{5 k T_{0} / 3 m}=\eta c
$$

bei den für die strahlenden Objekte in Frage kommenden Elektronentemperaturen $(1000 \mathrm{~km} / \mathrm{sec}$ entspricht $40000^{\circ} \mathrm{K}$ ). Obige Beobachtung deckt sich also mit dem in Arbeit A gefundenen Ergebnis, daß mit wesentlicher Ladungstrennung zu rechnen ist, wenn Strömungsgeschwindigkeiten auftreten, die mit der Schallgeschwindigkeit vergleichbar sind, was dann nach dem Mechanismus der vorliegenden Arbeit zu Radiostrahlung führen muß. Wir werden also für $\mathrm{Ab}$ schätzungszwecke $v_{\mathrm{L}}=\bar{v}=\varepsilon c=a_{\mathrm{e}}=\eta c$ setzen.

Eine Prüfung, welche Intensitäten unser Mechanismus zu liefern imstande ist, werden wir nun nicht bei dem oben erwähnten Maximum des Strahlungsstromes vornehmen, da der zugehörige Wert des Brechungsindex so nahe bei Null liegt, daß er bei vorhandener Dämpfung möglicherweise gar nicht erreicht werden kann. Vielmehr wählen wir zu dem eben vorgenommenen Ansatz $\varepsilon=\eta$ den nach der Dispersionsformel zu genau der Plasmafrequenz $(\alpha=1)$ gehörigen Wert $n=\varepsilon / \eta=1=C$ (Pseudovakuumwelle, s. §4). Damit erhält man für die Größenordnung des zu erwartenden Strahlungsstromes nach (30) am Entstehungsort die einfachen Ausdrücke

$$
S_{x} \simeq N_{0} \frac{m}{2} \frac{v^{4}}{c}-\frac{25}{18} \frac{N_{0}}{m c}\left(k T_{0}\right)^{2} .
$$

9 Siehe etwa zusammenfassende Darstellung von $\mathrm{H}$. Siedent opf, Z. angew. Phys. 6, 376 [1954] oder F. $\mathrm{Hoyle} \mathrm{e}^{10}$.
Einsetzen von $\bar{v}=1000 \mathrm{~km} / \mathrm{sec}$ führt bei $N_{0}=10^{8}$ (innere Sonnenkorona, $\omega_{\mathrm{e}}=2 \pi \cdot 100 \mathrm{MHz}$ ) zu $S_{x}=10^{-5} \mathrm{~W} / \mathrm{cm}^{2} \mathrm{sec}$, was etwa dem Strahlungsstrom eines $10 \mathrm{~kW}$-Senders in $100 \mathrm{~m}$ Abstand entspricht.

Die Radioastronomie gibt die Intensitäten vielfach durch Äquivalenttemperaturen $T_{\text {aeq }}$ an, die unter der Annahme berechnet werden, die strahlenden Objekte verhielten sich innerhalb des beobachteten Frequenzintervalls $\Delta v$ wie rein thermische schwarze Strahler nach der im Radiofrequenzgebiet anwendbaren Rayleigh-Jeansschen Strahlungsformel für polarisierte isotrope Emission

$$
I=\frac{v^{2}}{c^{2}} k T_{\text {aeq }} \Delta v .
$$

Die die Flächeneinheit senkrecht durchsetzende Komponente $\pi I$ des gesamten die Fläche in der Zeiteinheit passierenden Strahlungsflusses ist nun mit unserem Strahlungsstrom zu vergleichen, wobei wir für $\Delta v$ die üblichen Empfängerbandbreiten $(\simeq 1$ bis $20 \mathrm{MHz}$ ) und für $2 \pi v$ die Plasmafrequenz

$$
\omega_{\mathrm{e}}=\sqrt{4 \pi e^{2} N_{0} / m}
$$

einzusetzen haben. Bis auf unwesentliche Zahlenfaktoren ergibt sich daraus die Beziehung

$$
\frac{c k}{e^{2} \Delta v} T_{0}^{2}=T_{\mathrm{aeq}}
$$

oder zahlenmäßig mit $\Delta v=20 \mathrm{MHz}$

$$
10^{6} T_{0}^{2}=T_{\text {aeq }},
$$

wobei das Gleichheitszeichen in (35) selbstverständlich nur im Sinne eines ganz groben Größenordnungsvergleichs zu verstehen ist.

Die beobachteten Äquivalenttemperaturen reichen bis zu $10^{15}{ }^{\circ} \mathrm{K}$ und mehr (solare Strahlungsausbrüche). Wie man an (35 a) sieht, macht die Erklärung solcher $T_{\text {aeq }}$-Werte und der damit verbundenen überthermischen Radiostrahlungsintensitäten mit Hilfe unseres Mechanismus keinerlei Schwierigkeiten und es braucht dabei nicht zu sehr hohen, wenig plausiblen Elektronentemperaturen gegriffen zu werden, wie sie in der Literatur teilweise als real angesehen werden ${ }^{11}$. Wir haben dabei zu Abschätzungen nach der (35) zugrunde liegenden Art noch genügend Reserven und Spielraum zur Verfügung, um alle möglichen Effekte in Rechnung zu stellen, die zu kleineren oder größeren Strahlungsströmen führen könnten, wie $v_{\mathrm{L}}, \bar{v} \lessgtr a_{\mathrm{e}}, n \lessgtr 1, \omega>\omega_{\mathrm{e}}$,

10 F. H o y l e, Nature, Lond. 172, 296 [1953].

11 Zum Beispiel: M. R y l e, Proc. Phys. Soc., Lond. (A) 62, 491 [1949] oder F. H o y l e, l. c. ${ }^{10}$. 
ferner Absorption oder die Tatsache, daß unsere Abschätzung von der quasistationären Betrachtung des monochromatisch eingeschwungenen Plasmas mit langen Wellenzügen ausgeht. Zum Beispiel ergäbe sich mit $n<1$ eine Steigerung des Strahlungsstromes, die in der Gegend des zu Eingang des Paragraphen erwähnten Maximums von $n^{3} / C^{2} \alpha^{2}$ einen Faktor von der Größenordnung $c / 2 \bar{v}$ ausmachen würde.

\section{§ 7. Ergänzende Bemerkungen}

Bei der Strahlungsstrom-Abschätzung im vorhergehenden Paragraphen haben wir Brechungsindexwerte der Umgebung von 1 zugrunde gelegt. Wie wir schon ausgeführt haben $(\S 3)$, können dann die Dichteschwankungen nur klein sein, wenn man nicht mit der Lichtgeschwindigkeit vergleichbare Elektronengeschwindigkeiten

$$
v_{\mathrm{L}}=\frac{c}{n}\left|w_{1}-1\right|=\frac{c}{n}\left|\frac{N_{0}}{N_{\mathrm{m}}}-1\right|
$$

zulassen will. Da sich unter diesen Bedingungen bereits völlig ausreichende Strahlungsströme ergaben, um die beobachteten überthermischen Intensitäten zu erklären, erscheint es aussichtsvoll, zur Beschreibung von Entstehung und Verhalten allgemeinerer elektromagnetischer Strahlungsfelder in kosmischen Plasmen die magneto-hydrodynamischen Gleichungen für ein kompressibles Medium heranzuziehen, die der Verfasser in einer vorangegangenen Arbeit ${ }^{12}$ unter Berücksichtigung von Ladungstrennung und Dichteschwankungen kleineren Ausmaßes abgeleitet hat, wobei nur zwei variable Größen in den Gleichungen auftreten. Eine solche allgemeinere Behandlung mag wünschenswert erscheinen, da die vorliegende Arbeit, wie schon bemerkt, nur den quasistationär monochromatisch eingeschwungenen Plasmazustand erfaßt.

Allerdings scheint der Zustand des monochromatisch schwingenden Plasmas in der Natur wohl wegen der in $\S 5$ erwähnten Bevorzugung der Plasmafrequenz mit sehr großer Näherung tatsächlich realisiert zu sein, wie man dies aus dem Aussehen des Frequenzspektrums von Strahlungsausbrüchen schließen muß. Die beobachteten Frequenzbandbreiten im Meterwellenbereich betragen oft nur wenige Prozent ${ }^{13}$ und sind damit etwa gleich derjenigen von Fernsehsendern, so daß ein einzelner Wellenzug schon einige 10 Wellenlängen umfassen muß.

Gelegentlich wird bei einem Strahlungsausbruch auch die zweite Harmonische der Plasmafrequenz beobachtet, wie erstmalig Wild und Mitarb. ${ }^{13}$ mitgeteilt haben. Es muß sich also um anharmonische Wellen handeln. Für größere Dichteamplituden ergibt aber unser Mechanismus ein solches anharmonisches Verhalten, wie wir bereits in der Arbeit A erwähnten. (Siehe auch Abb. 3!) Damit sich größere Dichteamplituden quasistationär ausbilden können, muß die Fortpflanzungsgeschwindigkeit $v_{0}$ einerseits klein gegen die Lichtgeschwindigkeit, andererseits aber auch größer als die Elektronenschallgeschwindigkeit $a_{\mathrm{e}}=\eta c$ sein, wie wir in A gezeigt haben. Der Brechungsindex $n$ ist also für große Dichteamplituden an die Bedingung $1 \ll n<1 / \eta$ gebunden, so daß die Lösungen der Differentialgleichungen hier wesentlich im Bereich der speziellen Lösung II ( $\$ 3$ ) liegen. Wir werden daher die FourierKoeffizienten von $E_{z}$ bzw. $H_{y} \sim u^{\prime}$ für diesen Lösungsbereich berechnen, wobei wir der Einfachheit halber den Druckeinfluß vernachlässigen, also

$$
1 \ll C \simeq n^{2} \ll 1 / \eta^{2} \quad \text { und } \quad w_{1} \gg w_{\mathrm{g}} \simeq(\eta n)^{3 / 4}
$$

sowie $\varepsilon^{2} \ll 1$ und $\alpha \simeq 1$ voraussetzen.

Nach (29) und (13) gilt dann $u^{\prime} \sim \frac{w^{2 \prime}}{2} \simeq \pm \sqrt{\left(w_{1}-1\right)^{2}-(w-1)^{2}}, \quad u^{\prime \prime} \sim \frac{w^{2 \prime \prime}}{2} \simeq \frac{1}{w}-1$

mit $w(\xi)$ nach $(21) \quad \pm\left(\xi-\frac{\pi}{2}\right) \simeq \arcsin \frac{w-1}{\left|w_{1}-1\right|}-\sqrt{\left(w_{1}-1\right)^{2}-(w-1)^{2}}$.

Es ist einfacher, die Fourier-Koeffizienten von $u^{\prime}$ nicht unmittelbar zu bestimmen, sondern zunächst diejenigen von $u^{\prime \prime}$ zu berechnen und dann durch Integration auf $u^{\prime}$ überzugehen. Schreiben wir

$$
\frac{w^{2 \prime \prime}}{2}=\frac{1}{w}-1=\sum_{\mu} \mu A_{\mu} \cos \mu \xi, \quad u^{\prime} \sim \sum_{\mu} A_{\mu} \sin \mu \xi,
$$

12 R. W. Larenz, Z. Naturforschg. 10a, 761 [1955].

13 J. P. Wild, J. D. Murray u. W. C. Rowe, Nature, Lond. 172, 533 [1953]. 
so sind die Integrale der Form

$$
\mu A_{u}=\frac{1}{\pi} \int_{-\pi}^{+\pi}\left(\frac{1}{w}-1\right) \cos \mu \xi \mathrm{d} \xi=\frac{2}{\pi\left|w_{1}-1\right|} \int_{w_{1}}^{2-w_{1}} \frac{1}{w} \cos \mu\left\{\frac{\pi}{2}+\arcsin \frac{w-1}{\left|w_{1}-1\right|}-\left|w_{1}-1\right| \sqrt{\left.1-\left(\frac{w-1}{w_{1}-1}\right)^{2}\right\}} \frac{w \mathrm{~d} w}{\sqrt{1-\left(\frac{w-1}{w_{1}-1}\right)^{2}}}\right.
$$

zu berechnen, die durch die Substitution $w=1+a \sin \chi, \quad\left|w_{1}-1\right|=a$

übergehen in $\mu A_{\mu}=\frac{4}{\pi} \cdot(-1)^{\frac{\mu-1}{2}} \cdot \int_{0}^{\frac{\mu}{2}} \cos \mu \chi \cdot\left\{\int_{\cos }^{\sin }(\mu a \cos \chi)\right\} \mathrm{d} \chi$ für $\mu$ ungerade gerade.

Mit Hilfe von Entwicklung von $\sin (\mu a \cos \chi)$ nach Potenzen von $a \cdot \cos \chi$ und Anwendung der trigonometrischen Additionstheoreme lassen sich die Integrale leicht berechien. Es ergibt sich für die ersten 4 Koeffizienten:

$$
\begin{array}{ll}
A_{1}=a\left\{1-\frac{a^{2}}{8}+\frac{a^{4}}{192}-\frac{a^{6}}{36 \cdot 256}+\ldots\right\}, & A_{2}=\frac{1}{2} a^{2}\left\{1-\frac{a^{2}}{3}+\frac{a^{4}}{24}-\frac{a^{6}}{360}+\ldots\right\}, \\
A_{3}=\frac{3}{8} a^{3}\left\{1-\frac{9}{16} a^{2}+\frac{81}{640} a^{4}-\frac{81}{5120} a^{6}+\ldots\right\}, & A_{4}=\frac{1}{3} a^{4}\left\{1-\frac{4}{5} a^{2}+\frac{4}{15} a^{4}-\frac{16}{315} a^{6}+\ldots\right\} .
\end{array}
$$

\section{Im Grenzfall}

$$
\left|w_{1}-1\right|=a \rightarrow 1 \quad\left[\text { aber } w_{1}>(\eta n)^{3 / 4}\right]
$$

erhalten wir für das Intensitätsverhältnis der Harmonischen

$$
A_{1}^{2}: A_{2}^{2}: A_{3}^{2}: A_{4}^{2} \rightarrow 1: 0,16: 0,054: 0,025 \text {. }
$$

Besonders die 2. Harmonische erscheint also schon bei mäßigen Amplituden mit einer Intensität, die derjenigen der Grundwelle durchaus vergleichbar ist $^{14}$. Bei Berücksichtigung des Elektronendrucks steigt der Harmonischenanteil noch etwas. Tatsächlich wird die 2. Harmonische oft mit größerer Intensität als die Grundwelle beobachtet, was darauf zurückzuführen ist, daß die Grundwelle ( Plasmafrequenz) bei ihrer weiteren Ausbreitung vom Entstehungsort aus stärker gedämpft wird als höhere Frequenzen, was man im einzelnen bei $\mathrm{W}$ ild ${ }^{13}$ nachlesen möge.

Wir bemerken noch, daß für solche Wellen großer Amplitude bei relativ kleiner Fortpflanzungsgeschwindigkeit $\left(n^{2} \gg 1\right)$ die Wellenlängen am Entstehungsort natürlich sehr viel kleiner sein müssen als die Vakuumwellenlängen bei der gleichen Frequenz.

Für die Anregung der Plasma-Schwingungen bzw. -Wellen halten wir folgenden Mechanismus für möglich: Beim Fortschreiten einer Stoßwelle im Plasma läuft der eigentlichen,

14 In der Umgebung von $n=1$ würde bei entsprechend großen Elektronengeschwindigkeiten ${A_{2}}^{2}: A_{1}{ }^{2} \cong 0,4$ sein. im wesentlichen aus der massereichen Ionenkomponente gebildeten Stoßfront eine negative Elektronenraumladung voraus, wie wir dies in Arbeit A für die ,aperiodische Ionenschallwelle" gefunden haben, die man als adiabatisch reversiblen Grenzfall einer allgemeinen Plasmastoßwelle ansehen kann. Übersteigt nun die Stoßwellenfrontgeschwindigkeit

$$
v_{0}^{2}=\frac{5 k T_{0}}{3\left(m_{\mathrm{i}}+m_{\mathrm{e}}\right)} \frac{6}{4 / d-1}
$$

( $d=$ Verdichtung, $\quad m_{\mathrm{i}, \mathrm{e}}=$ Ionen- bzw. Elektronenmasse) die Elektronenschallgeschwindigkeit $\left(a_{\mathrm{e}}^{2}=5 k T_{0} / 3 m_{\mathrm{e}}\right)$ vor der Stoßfront, so wird die für kleine Stoßgeschwindigkeiten monotone, im Koordinatensystem der Stoßwelle im statischen Gleichgewicht befindliche Raumladungsverteilung vor der Front nach unserer Vermutung instabil und gliedert sich (durch "Aufschiebung“) in Verdichtungen und Verdünnungen, eben die Plasmaschwingungen auf.

Da die Plasmafrequenz offenbar eine bevorzugte Stellung einnimmt, müssen wir annehmen, daß das Frequenzspektrum einer Strahlungsquelle oder einer Gesamtheit von Quellen mit statistischer Emission überthermischer Radiostrahlung sich im wesentlichen aus den Beiträgen der Gebiete verschiedener Dichte zusammensetzt, da letztere ja die Plasmafrequenz bestimmt. Das Spektrum kann daher je nach Aufbau der Strahlungsquelle und deren Aktivität in den einzelnen Gebieten ganz verschieden aussehen. Der allgemein beobachtete Abfall nach hohen Frequenzen hin dürfte durch einen mit der Dichte und daher mit der Frequenz anwachsenden Dämpfungseffekt bedingt sein, der etwa mit dem Abfall des hydrodynamischen Turbulenzspektrums verglichen werden kann.

Herrn Prof. G. B u rkha rdt danke ich für wertvolle Diskussionen. 